\title{
Fisiopatologia del rigetto acuto e cronico
}

Giornale di Tecniche Nefrologiche e Dialitiche 2019, Vol. 31(2) 128-132

(C) The Author(s) 2019

Article reuse guidelines:

sagepub.com/journals-permissions

DOI: $10.1177 / 0394936219847416$

journals.sagepub.com/home/gtn

\section{(S)AGE}

\author{
Aris Tsalouchos' e Maurizio Salvadori²
}

\begin{abstract}
Physiopathology of acute and chronic rejection

Allograft rejection is defined as tissue injury produced by the effector mechanisms of the alloimmune response, leading to deterioration of graft function. There are two types of rejection: T-cell-mediated rejection (TCMR) and antibodymediated rejection (AMR). Both types of rejection can be early or late, fulminant or indolent, as well as isolated or concomitant and can share pathologic features on biopsy.

The immune response to an allograft is an ongoing dialogue between the innate and adaptive immune system, which if unresolved will lead to rejection of the transplanted cells, tissues, or organs. Activation of elements of the innate immune system, triggered by tissue injury sustained during cell isolation or organ retrieval and ischemia reperfusion, will initiate and amplify the adaptive response. T cells require a minimum of two signals for activation: antigen recognition and co-stimulation.

Antibody-mediated rejection triggered by alloantibody binding and complement activation is increasingly recognized as a significant contribution to graft loss. Even though one component of the immune system may dominate and lead to rejection, this is usually multifactorial, resulting from the integration of multiple mechanisms. Identifying the molecular pathways that trigger rejection facilitates the identification of targets for the development of immunosuppressive drugs.
\end{abstract}

\section{Keywords}

Acute rejection, Chronic rejection, Antibody-mediated rejection, Cellular components of rejection, Complement cascade

\section{Introduzione}

Il rigetto del trapianto renale può essere definito come il danno inferto all'organo trapiantato dai meccanismi effettori della risposta immune dei quali è stato parlato nella rassegna precedente.

Esistono due tipi di rigetto: il rigetto mediato dalle cellule $\mathrm{T}$ (T-cell-mediated rejection, TCMR) ed il rigetto mediato dagli anticorpi (antibody-mediated rejection, AMR). Entrambi possono essere precoci o tardivi nel decorso post-trapianto, con decorso clinico acuto o cronico.

\section{Reclutamento delle cellule nell'interstizio del trapianto}

Gli elementi della risposta immune che causano rigetto sono cellulari ed umorali. Le cellule coinvolte sono i linfociti T, i macrofagi, i linfociti B, le plasmacellule, gli eosinofili ed i leucociti neutrofili. Le strutture bersaglio sono prevalentemente l'endotelio e le cellule tubulari.
I linfociti $\mathrm{T}$ sono le cellule effettrici principalmente coinvolte, spesso coadiuvati dai macrofagi. I linfociti B e le plasmacellule servono per la produzione di anticorpi. I neutrofili producono danno alle strutture bersaglio con la produzione di chemochine.

Le cellule effettrici penetrano nel trapianto seguendo tre fasi: rallentamento leucocitario lungo la parete capillare, adesione alla parete capillare e migrazione attraverso la parete capillare.

\footnotetext{
'Azienda UsI Toscana Centro, S.O.S Nefrologia e Dialisi, Ospedale SS. Cosma e Damiano, Via Cesare Battisti 2, 51017 Pescia, Italy 2Professore di Nefrologia già Direttore Nefrologia e Trapianto, Azienda Ospedaliera Careggi, Viale Pieraccini I8, 50I39 Firenze, Italy

Corrispondenza:

Professor Maurizio Salvadori, Professore di Nefrologia già Direttore Nefrologia e Trapianto, Azienda Ospedaliera Careggi, Via Massicini I5, 50014 Fiesole Firenze, Italy.

Email: maurizio.salvadoril@gmail.com
} 
Tabella I. Proteine coinvolte nel reclutamento dei leucociti nel trapianto.

\begin{tabular}{|c|c|c|c|}
\hline Proteina & Nome & Ligando & Funzione \\
\hline Selettine & $\begin{array}{l}\text { CD62L (L-selettina) } \\
\text { CD62L (P-selettina) } \\
\text { CD62L (E-selettina) }\end{array}$ & $\begin{array}{l}\text { Glicoproteine legate } \\
\text { all'acido sialico }\end{array}$ & Adesione dei leucociti all'endotelio \\
\hline Chemochine & $\begin{array}{l}\text { MCP-I/CCL2 } \\
\text { MIP-I } \alpha / C C L 3 \\
\text { RANTES/CCL5 } \\
\text { IL-8/CXCL8 } \\
\text { MIG/CXCL9 } \\
\text { IP-I0/CXCLI0 } \\
\text { Lymphotactina/XCLI }\end{array}$ & $\begin{array}{l}\text { CCR2 } \\
\text { CCRI } \\
\text { CCRI, CCR4, CCR5 } \\
\text { CXCRI, CXCR2 } \\
\text { CXCR3 } \\
\text { CXCR3 } \\
\text { XCRI }\end{array}$ & $\begin{array}{l}\text { Reclutamento di monociti, cellule } \\
\text { dendritiche immature, cellule T } \\
\text { Reclutamento di monociti, cellule } \\
\text { dendritiche immature, cellule T } \\
\text { Reclutamento di monociti, cellule } \\
\text { dendritiche, cellule T, cellule NK } \\
\text { Reclutamento di neutrofili } \\
\text { Reclutamento di cellule T della memoria } \\
\text { Reclutamento di cellule T della memoria } \\
\text { Reclutamento di cellule T }\end{array}$ \\
\hline $\begin{array}{l}\text { Superfamiglia } \\
\text { delle } \\
\text { immunoglobuline }\end{array}$ & $\begin{array}{l}\text { CD54/ICAM-I } \\
\text { CDI02/ICAM-2 } \\
\text { CD50/ICAM-3 } \\
\text { CDI06/VCAM-I } \\
\text { CD3I/PECAM-I }\end{array}$ & $\begin{array}{l}\text { LFA-I } \\
\text { LFA-I } \\
\text { VLA-4 } \\
\text { CD3I }\end{array}$ & $\begin{array}{l}\text { Lieve adesione dei leucociti all'endotelio } \\
\text { Lieve adesione dei leucociti all'endotelio } \\
\text { Lieve adesione dei leucociti all'endotelio } \\
\text { Stravaso dei leucociti attraverso l'endotelio }\end{array}$ \\
\hline
\end{tabular}

Questi processi sono mediati da proteine chiamate citochine e chemochine. La tabella 1 elenca queste sostanze e ne evidenzia il meccanismo d'azione.

Le selettine provocano un rallentamento dei leucociti che, in tal modo, allungano i tempi di contatto con la parete endoteliale. In questo modo le cellule vengono più facilmente stimolate dalle chemochine che hanno il loro recettore sulla parete dei leucociti. La leukocyte functionassociated antigen (LFA-1) è un'integrina espressa sulla maggioranza dei leucociti. I ligandi per la LFA, come le intercellular adhesion molecule (ICAM) 1, 2 e 3, sono debolmente espressi sulla parete endoteliale, ma possono essere potentemente attivati da citochine come interleuchina 1 (IL-1) e tumor necrosis factor $\alpha$ (TNF- $\alpha)$. Di conseguenza i leucociti aderiscono in modo forte alla parete, riconoscono l'antigene, si muovono attraverso la parete e secernono proteasi che degradano la membrana basale e favoriscono la migrazione nell'interstizio. Qui le cellule $\mathrm{T}$, già attivate, riconoscono l'antigene estraneo, rilasciano citochine pro-infiammatorie o distruggono direttamente le cellule esogene.

\section{Rigetto acuto mediato da cellule $\mathbf{T}$}

A creare il danno al trapianto concorrono sia l'immunità adattiva che l'immunità innata. L'immunità adattiva è strettamente dipendente dall'attivazione e differenziazione delle cellule $\mathrm{T}$ che hanno riconosciuto l'antigene estraneo (figura 1). ${ }^{1}$

Le cellule Th1 rilasciano interferon- $\gamma$ e IL-2, che favoriscono sia l'attivazione cellulare, sia la produzione di anticorpi. Le cellule Th17 producono la citochina proinfiammatoria IL-17 che media la flogosi sia stimolando altre citochine, sia favorendo il reclutamento di neutrofili e macrofagi. ${ }^{2}$ In contrasto ai Th1 e Th17, i linfociti Th2 producono citochine antiinfiammatorie come IL-10 e IL-4. Le cellule Treg così prodotte prevengono la produzione di anticorpi e favoriscono la tolleranza. ${ }^{3}$

L'immunità innata è coinvolta nel rigetto cellulare. I macrofagi sono coinvolti ed agiscono come cellule presentanti l'antigene alle cellule T. Sempre i macrofagi si legano agli anticorpi prodotti dalle cellule B e sviluppano la tossicità cellulare anticorpo-dipendente.

Le cellule natural killer (NK) contribuiscono al danno tessutale ed hanno la capacità di uccidere le cellule bersaglio. ${ }^{4}$

Anche i neutrofili sono implicati e mediano il danno dei tessuti producendo le chemochine CXCL1, 2, 3 e 8 e producendo radicali di ossigeno.

Infine, i macrofagi che sono richiamati dalle citochine IL-1 ed IL-6 producono enzimi capaci di degradare sia l'endotelio vascolare che il parenchima. ${ }^{5}$

Nel rigetto cellulare è infine attivata anche la cascata complementare in grado di facilitare il passaggio dalla risposta immune innata a quella adattiva. ${ }^{6}$

Aspetti caratteristici del rigetto acuto mediato da cellule sono la tubulite e l'infiltrazione dell'epitelio tubulare da parte delle cellule $\mathrm{T}$ e di cellule mieloidi come i monociti, i macrofagi e le cellule dendritiche. Il deterioramento della funzione renale durante il TCMR acuto è correlato con la gravità della tubulite e della flogosi vascolare che è però meno frequente.

I linfociti T possono uccidere le cellule bersaglio tramite il rilascio di molecole citotossiche come la perforina, il granzyme B e la granulolisina.

Alcuni studi suggeriscono che la tubulite può coinvolgere un subset specifico di cellule $\mathrm{T}$ che esprimono l'integrina CD103 che si lega al suo ligando e-caderina sulle cellule epiteliali. Tuttavia, studi sugli animali hanno 


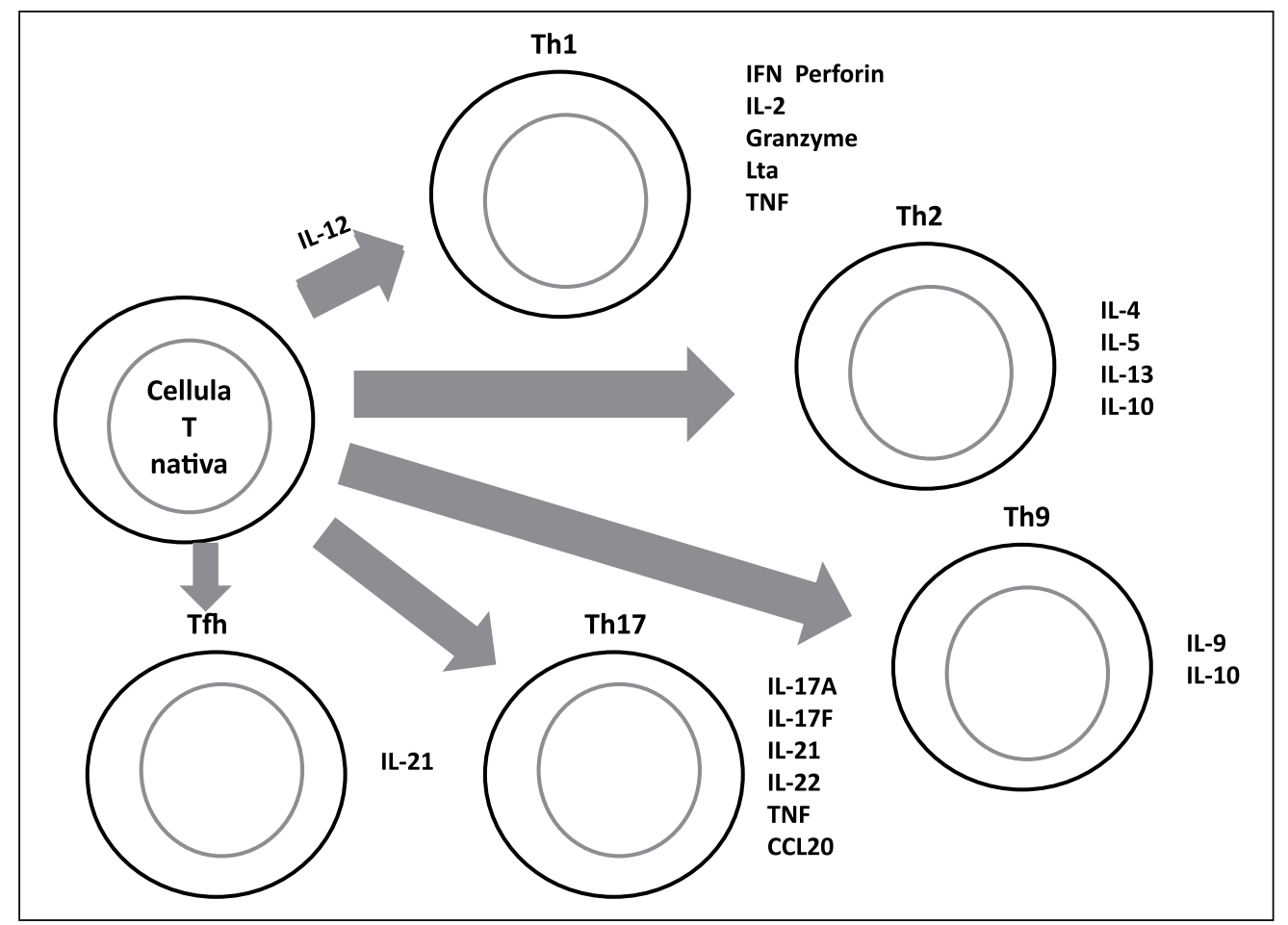

Figura I. Differenziazione delle cellule T dopo riconoscimento dell'antigene.

documentato che il rigetto acuto avviene anche in assenza di perforina/granzyme, Fas-FasL e CD103. ${ }^{7}$

Le cellule $\mathrm{T}$ potrebbero mediare rigetto attraverso la secrezione di citochine, tramite il loro effetto diretto, o tramite la loro capacità di attivare i macrofagi e generare una risposta di tipo delayed-type hypersensitivity (DTH) ${ }^{8}$ La risposta DTH genera rilascio di ossigeno reattivo, enzimi proteolitici, eicosanoidi. Meno frequentemente si osserva endoarterite che sembra essere indipendente dalle lesioni tubulo-interstiziali. La glomerulite è eccezionale ed in tal caso le cellule infiltranti sono cellule T e macrofagi.

\section{Rigetto acuto mediato da anticorpi}

Si tratta di un rigetto acuto causato da anticorpi antidonatore, spesso causa di perdita dell'organo. Si distingue un AMR iperacuto ed un AMR acuto. La forma iperacuta è sempre dovuta ad anticorpi preformati, presenti a titolo elevato nel pretrapianto. Si tratta di un evento raro perché le tecniche in uso permettono di evidenziare tali anticorpi.

La forma acuta è anch'essa dovuta quasi sempre ad anticorpi anti-HLA del donatore, ma non evidenziabili prima del trapianto. Sono anticorpi diretti contro l'endotelio capillare e peritubulare. Il danno è soprattutto dovuto alla loro capacità di legare ed attivare il complemento. Può verificarsi congiuntamente con una componente di rigetto cellulo-mediato.

Come detto, quasi sempre sono anticorpi diretti contro il sistema HLA del donatore, ma esistono anche AMR

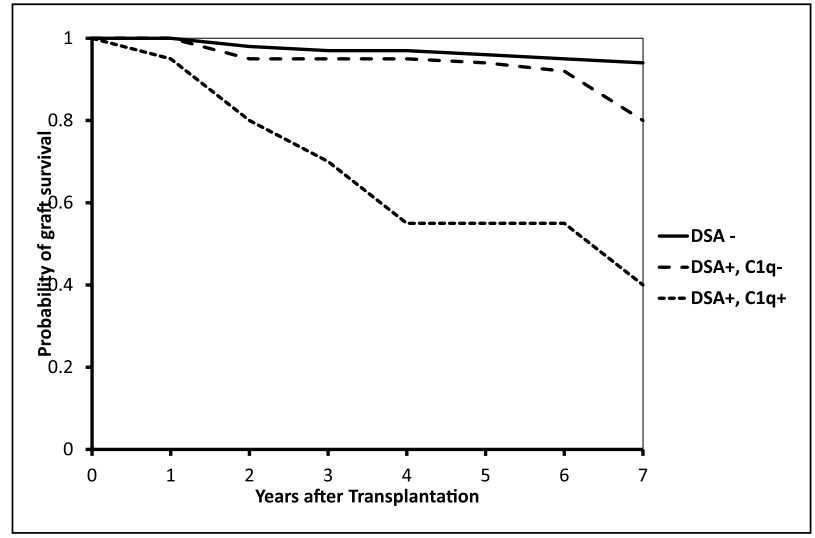

Figura 2. Peggior sopravvivenza se anticorpi anti-DSA fissano il Clq.

dovuti ad anticorpi diretti contro altre strutture come gli antigeni del gruppo ematico $\mathrm{AB} 0$. In casi più rari si tratta di autoanticorpi diretti contro il recettore di tipo I dell'angiotensina II o altri antigeni dell'endotelio.

Il rene mostra accumulo di cellule $T$, neutrofili e monociti nei capillari peritubulari e glomerulari. La tubulite è in genere lieve.

La tossicità ed il potere lesivo degli anticorpi è dovuto alla loro capacità di attivare il complemento, in genere per la via classica. Questo è ben documentato dall'assenza di danno, anche in presenza di anticorpi, se il C1q è bloccato (figura 2). 


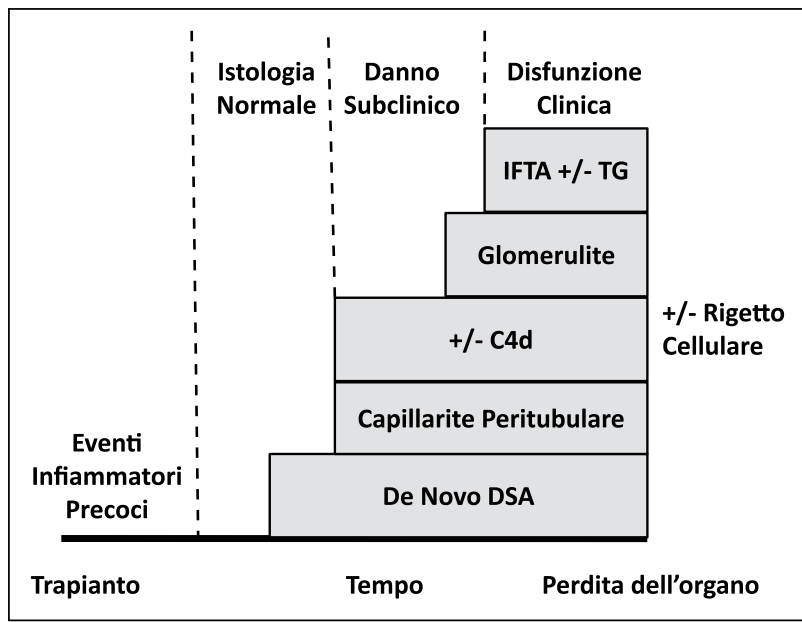

Figura 3. Storia naturale del danno inferto dagli anticorpi.

Generalmente gli anticorpi fissano il C1q ed attivano il C3. Questo si scinde in C3a e C3b ed il C3b amplifica la cascata complementare. Le anafilotossine C3a e C5a sono potenti nell'attrarre neutrofili e macrofagi. $\mathrm{Si}$ ha vasospasmo attraverso il rilascio di prostaglandina E2 da parte dei macrofagi ed edema attraverso il rilascio di istamina da parte dei granulociti basofili. C3a e C5a incrementano le molecole di adesione endoteliali e favoriscono il rilascio di diverse citochine e chemochine. Infine il membrane attack complex (MAC), C5b-C9, causa lisi della cellula endoteliale. Gli anticorpi possono anche dare danno cellulare in modo indipendente dal complemento, reclutando i leucociti attraverso il recettore $\mathrm{Fc}$ (più spesso il CD16), coinvolgendo le cellule NK ed i macrofagi (la tossicità cellulare anticorpo-dipendente). Il risultato finale è un'alterazione irreversibile della membrana basale, formazione di microtrombi e rilascio del fattore von Willebrand che determina aggregazione piastrinica. ${ }^{9}$

L'attivazione del complemento è documentata dalla presenza nei capillari peritubulari del $\mathrm{C} 4 \mathrm{~d}$. Infatti, la diagnosi di AMR si basa su tre parametri: 1) l'evidenza morfologica di un danno acuto;2) l'evidenza immunologica di un'attività anticorpale con la deposizione di $\mathrm{C} 4 \mathrm{~d} ; 3$ ) l'evidenza nel siero di anticorpi anti-HLA o diretti verso altri antigeni endoteliali. ${ }^{10}$

È infine importante ricordare che anticorpi anti-HLA, oltre a generare AMR, possono, in maniera importante, contribuire a determinare danno a distanza di tempo nell'ambito del cosiddetto rigetto cronico (figura 3 ).

\section{Rigetto cronico}

Si tratta della lenta e progressiva perdita di funzione dell'organo trapiantato. A questo fenomeno possono concorrere differenti cause. Nell'insieme si chiama nefropatia cronica del trapianto. Per rigetto cronico si deve intendere il danno inferto da cause immunologiche (figura 4). Una serie di studi hanno documentato che il vero rigetto cronico è responsabile della perdita del $50 \%$ degli organi trapiantati, mentre il resto è dovuto a fenomeni come tossicità da inibitori delle calcineurine, ipertensione, infezioni come quelle da virus BK, complicanze chirurgiche, recidiva della malattia primitiva.

Fra i fattori non immunologici esistono quelli presenti prima del trapianto o al momento del trapianto stesso, come il danno da ischemia-riperfusione, danno sofferto dall'organo al momento del trapianto, morte cerebrale del donatore, età del donatore.

In molti casi questi fattori di per sé non immuni, possono attivare l'immunità innata, portando ad un'aumentata espressione degli antigeni del sistema maggiore di istocompatibilità, stimolando così l'immunità adattiva.

Si ritiene che gli stessi meccanismi effettori presenti nel rigetto acuto siano anche presenti nel rigetto cronico. L'evidenza di questo viene da un insieme di studi che hanno documentato che i peptidi derivanti dal sistema maggiore di istocompatibilità del donatore sono capaci di stimolare una risposta immune anche molto tempo dopo che sono scomparse dall'organo le cellule presentanti l'antigene. Anche gli anticorpi si ritiene che abbiano un'importanza di rilievo nello sviluppo del rigetto cronico.

Il rigetto cronico può essere dovuto sia a meccanismi cellulari sia umorali. Gli aspetti istologici tipici sono la glomerulopatia da trapianto, la capillaropatia peritubulare, l'arteriopatia e l'atrofia tubulare con fibrosi interstiziale.

Si ha duplicazione o multi-laminazione della membrana basale glomerulare, con espansione del mesangio e accumulo di cellule mononucleate.

Si ritiene che prevalga il danno mediato da anticorpi, poiché nella maggioranza delle glomerulopatie da trapianto si hanno anticorpi circolanti diretti contro gli antigeni del donatore della seconda classe del sistema maggiore d'istocompatibilità e spesso si ha deposizione di $\mathrm{C} 4 \mathrm{~d}$ nei capillari peritubulari.

L'arteriopatia da trapianto è caratterizzata da ispessimento dell'intima arteriolare nel cui interno sono visibili cellule $\mathrm{T}$ e macrofagi, espressione di attività immunitaria cellulo-mediata.

Gli attuali criteri per identificare un rigetto cronico attivo anticorpo-mediato sono l'evidenza istologica di un danno cronico, l'evidenza immunopatologica di un'attività anticorpale, e l'evidenza della presenza in circolo di anticorpi reagenti nei confronti del sistema HLA del donatore. Il primo evento è la produzione di anticorpi (stadio I), seguito dall'interazione fra anticorpi e antigene con deposizione di $\mathrm{C} 4 \mathrm{~d}$ nei capillari peritubulari e nei glomeruli (stadio II), seguito da alterazioni istologiche (stadio III) e finalmente l'alterazione funzionale del trapianto (stadio IV). 


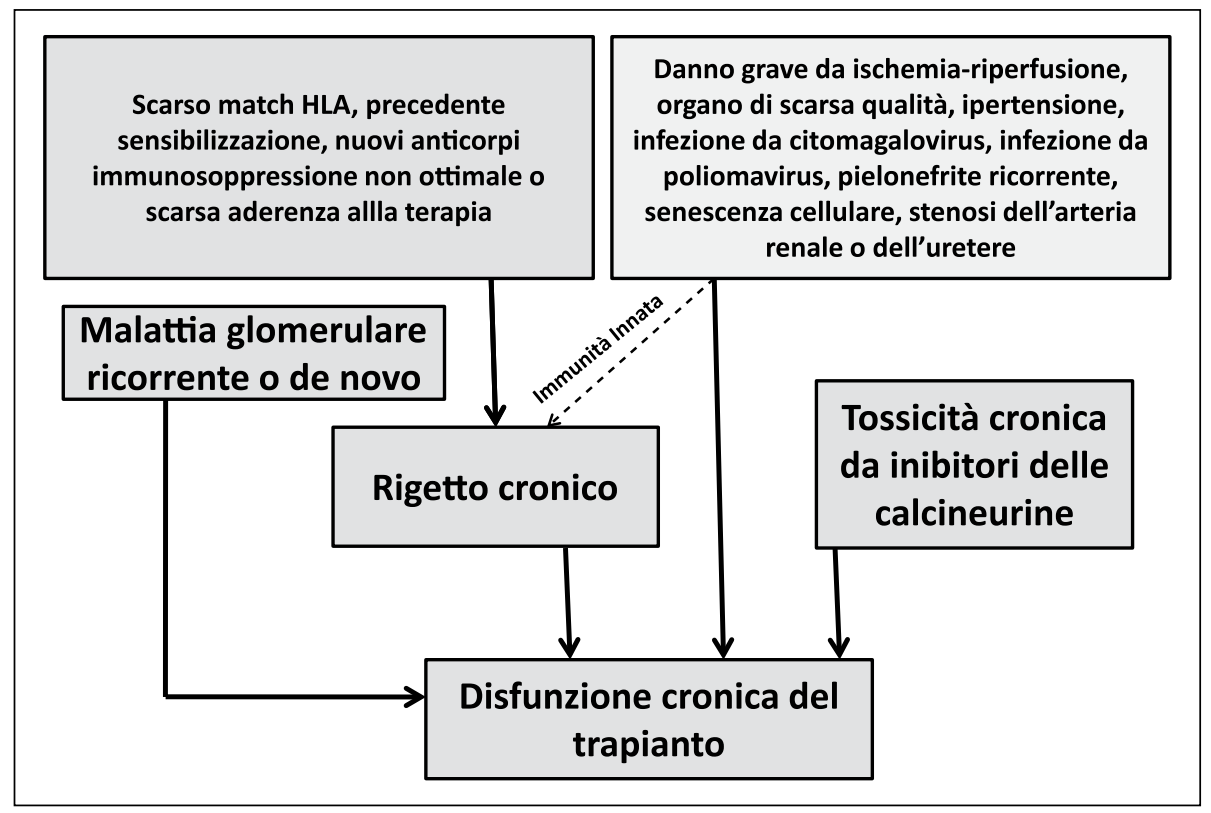

Figura 4. Meccanismi causali della nefropatia cronica da trapianto.

A parte il danno mediato dagli anticorpi in cui è coinvolto il complemento, diversi studi hanno delucidato altri meccanismi attraverso i quali è provocato danno al tessuto renale nel rigetto cronico.

Il TGF- $\beta$ può sviluppare fibrosi. La riduzione della fibrosi indotta dal TGF- $\beta$ può essere inibita da sostanze che inibiscono il TGF- $\beta$ come la decorina, il pirfenidone, la relaxina o la proteina morfogenetica dell'osso (Bmp7). Similmente agiscono l'angiotensina II e l'endotelina I. Un fenomeno di particolare interesse è la transizione epiteliomesenchimale. Secondo questo fenomeno, cellule residenti di differente natura come cellule mesenchimali o epiteliali possono trasformarsi in fibroblasti, portando alla fibrosi.

\section{Dichiarazione di assenza di conflitto di interessi}

Gli Autori dichiarano di non avere conflitti di interessi.

\section{Finanziamenti}

Gli Autori dichiarano di non aver ricevuto finanziamenti specifici da qualsiasi ente nei settori pubblico, privato o senza fini di lucro.

\section{Bibliografia}

1. Wood KJ and Goto R. Mechanisms of rejection: current perspectives. Transplantation 2012; 93(1):1-10.
2. Mills KH. Induction, function and regulation of IL-17producing T cells. Eur J Immunol 2008; 38(10):2636-2649.

3. Long E and Wood KJ. Regulatory T cells in transplantation: transferring mouse studies to the clinic. Transplantation 2009; 88(9):1050-1056.

4. Li XC. The significance of non-T-cell pathways in graft rejection: implications for transplant tolerance. Transplantation 2010; 90(10):1043-1047.

5. Kitchens WH, Chase CM, Uehara S, et al. Macrophage depletion suppresses cardiac allograft vasculopathy in mice. J Immunol 2005; 175(5):3424-3430.

6. Asgari E, Zhou W and Sacks S. Complement in organ transplantation. Curr Opin Organ Transplant 2010; 15(4):486-491.

7. Einecke G, Fairhead T, Hidalgo LG, et al. Tubulitis and epithelial cell alterations in mouse kidney transplant rejection are independent of CD103, perforin or granzymes A/B. Am J Transplant 2006; 6(9):2109-2120.

8. Halloran PF. T cell-mediated rejection of kidney transplants: a personal viewpoint. Am J Transplant 2010; 10(5):11261134.

9. Colvin RB, Hirohashi T, Farris AB, et al. Emerging role of B cells in chronic allograft dysfunction. Kidney Int Suppl 2010; (119):S13-17.

10. Feucht HE. Complement $\mathrm{C} 4 \mathrm{~d}$ in graft capillaries - the missing link in the recognition of humoral alloreactivity. Am J Transplant 2003; 3(6):646-652. 